\title{
High viscosity hydroxypropylmethylcellulose reduces postprandial blood glucose concentrations in NIDDM patients
}

\author{
Christos Reppas ${ }^{a}$, Constance H. Adair ${ }^{b}$, Jeffrey L. Barnett ${ }^{c}$, Rosemary R. Berardi ${ }^{d}$, \\ Dan DuRoss ${ }^{b}$, Sahar Z. Swidan ${ }^{d}$, Paul F. Thill ${ }^{d}$, Stephen W. Tobey, \\ Jennifer B. Dressman* ${ }^{d}$ \\ "Department of Pharmacy, University of Athens, Athens, Greece \\ ${ }^{b}$ General Clinical Research Center, "Department of Internal Medicine. "College of Pharmacy. The University of Michigan. \\ Ann Arbor, MI 48109-1065. USA \\ 'The Dow Chemical Company, Midland, MI, USA
}

(Received 28 June 1993; accepted 9 September 1993)

\begin{abstract}
The ability of high viscosity hydroxypropylmethylcellulose (HPMC) to reduce postprandial glucose concentrations was assessed in patients with non-insulin-dependent diabetes (NIDDM) and healthy volunteers. The study design consisted of a two-way crossover, single-dose administration of $10 \mathrm{~g}$ prehydrated high viscosity HPMC, or placebo, with a standard carbohydrate-rich meal. In patients with NIDDM, HPMC reduced blood glucose concentrations at the 60-, 75-, 90-, 120- and 150-min sampling intervals, with an average reduction in the maximum postprandial blood glucose concentration, $C_{\max }$, of $24 \%(P<0.05)$. The time at which the maximum concentration was reached, $T_{\max }$, remained unchanged. The area under the blood concentration versus time plot, $A U C_{0-6 \mathrm{~h}}$, was reduced by an average of $15 \%$ $(P<0.05)$. The blood concentration profile of insulin followed that of glucose. Concentrations were significantly lower than in the placebo phase only at the 120 -min sampling time, while pharmacokinetic parameters $\left(C_{\max }, T_{\max }\right.$ and $\left.A U C_{0-6 \mathrm{~h}}\right)$ were unchanged. These results suggest that alterations in the blood glucose profile are mediated by luminal events rather than by changes in hormonal response. In contrast to the NIDDM patients, neither the pharmacokinetic parameters nor the blood glucose concentrations at specific sampling times were significantly affected by the coadministration of HPMC in healthy volunteers. Overall, the results of this study suggest that HPMC may be a useful adjunct in the management of NIDDM.
\end{abstract}

Key words: Hydroxypropylmethylcellulose; Water soluble fibers; Non-insulin-dependent diabetes mellitus; Glucose tolerance; Nutrient absorption

\footnotetext{
* Corresponding author.
}

0168-8227/93/\$06.00 (c) 1993 Elsevier Scientific Publishers Ireland Lid. All rights reserved. SSDI 0168-8227(93)0809-U 


\section{Introduction}

Certain water-soluble fibers, such as guar and pectin, reduce the postprandial blood glucose response to carbohydrate-rich test meals [1-3] and oral glucose loads [4-6]. These effects have been demonstrated in both healthy and diabetic subjects [7]. Comparison of results between viscous and non-viscous guar has shown that the ability of guar to develop a high viscosity in aqueous solution is crucial to its ability to reduce postprandial glucose concentrations [5]. Like guar and pectin, hydroxypropylmethylcellulose (HPMC) can gencrate high viscosities in aqueous solution. HPMC is a semi-synthetic cellulose derivative widely used in pharmaceutical products as a tablet excipient and thickening agent. Recent studies [8] indicate that high viscosity HPMC is capable of improving glucose tolerance in dogs. Medium viscosity HPMC had a lesser effect, while low viscosity HPMC failed to significantly improve glucose tolerance. These results suggest that HPMC, like guar and pectin, modulates blood glucose concentrations via an increase in luminal viscosity.

The object of the current study was to determine whether high viscosity HPMC could attenuate the postprandial blood glucose response in humans, and to compare the response in patients with noninsulin-dependent diabetes mellitus (NIDDM) with that in healthy volunteers.

\section{Materials and methods}

A clinical study was conducted to assess the ability of high viscosity HPMC (Dow Chemical, Midland, MI) $[9,10]$, to attenuate postprandial elevation of glucose concentrations in two groups of subjects. The first group comprised 10 patients with NIDDM and the second group consisted of 10 healthy volunteers with mildly elevated serum cholesterol concentrations. This control group was chosen because it has been shown recently that high viscosity HPMC is effective in reducing serum cholesterol concentrations in those with mildly elevated cholesterol [11]. The study was conducted on a double-blinded randomised twoway crossover basis and consisted of two phases, a placebo and a test phase, which were separated by at least 1 week. All studies were conducted at the General Clinical Research Center (GCRC) of the University of Michigan Hospitals, after receiving approval from the Institutional Review Board for studies involving human subjects and from the Food and Drug Administration. Written informed consent was obtained from each subject prior to participation. In the case of NIDDM patients, written approval from each patient's primary physician was also obtained prior to entry into the study.

\subsection{Exclusion and inclusion criteria}

Subjects were excluded if there was a history of major health problems, gastrointestinal (GI) disease, regular use of antacids or other drugs which may affect GI function, or use of drugs which affect cholesterol concentrations (e.g., psyllium). Subjects were required to forego alcohol and tobacco for a total of 3 days before and throughout each study phase. Each subject underwent a general medical examination, including phlebotomy to assess electrolyte balance, renal and hepatic function, and blood morphology. Women of childbearing age were screened by blood test for pregnancy. Women on birth control pills were counseled to take this medication more than $3 \mathrm{~h}$ away from the study meal.

NIDDM patients were required to have been diagnosed at least 2 years prior to study entry. None had experienced a body weight fluctuation of greater than $5 \%$ within the past year. None were receiving insulin, and all were required to withdraw from their usual hypoglycemic medication for at least three elimination half-lives (to ensure $>90 \%$ washout) prior to each study day. Patients receiving chlorpropamide were excluded because of its long elimination half-life.

Entry criteria for non-diabetic volunteers included a total cholesterol concentration greater than $5.15 \mathrm{mmol} / 1(200 \mathrm{mg} \%)$ but less than 8.30 $\mathrm{mmol} / 1(320 \mathrm{mg} \%)$, a fasting triglyceride of less than $3.45 \mathrm{mmol} / 1(300 \mathrm{mg} \%)$ and an LDL cholesterol concentration greater than $4.15 \mathrm{mmol} / \mathrm{l}$ (160 $\mathrm{mg} \%$ ). Subjects whose body weight was not within $20 \%$ of ideal body weight (as determined by Metropolitan Life Insurance Co. actuarial tables, 1983) were excluded. Gender, age and weight of 
the subjects were matched in the two groups as closely as possible, without exceeding the weight limitations for non-diabetic volunteers. A summary of subject demographics is presented in Table 1. Apart from fasting glucose $(P=0.0002)$ and total cholesterol $(P=0.0035)$, an unpaired $t$ test showed no difference (at the 0.05 level) between NIDDM and healthy subjects in the parameters listed in Table 1.

\subsection{Study protocol}

For a period of 3 days before each phase, all subjects were instructed to maintain their carbohydrate consumption at approximately $60 \%$ of the total caloric intake and to complete a diary of the type and quantity of food consumed. Subjects fasted from food but not water after 20:00 h the evening before the study day of each phase. In the test phase of the study, $10 \mathrm{~g}$ of HPMC was administered in prehydrated form as a diet Jello formulation, at a concentration of $2 \%$ (weight in volume) resulting in a volume of $500 \mathrm{ml}$. The placebo formulation consisted of $500 \mathrm{ml}$ of diet Jell- $\mathrm{O}^{\oplus}$ in which one tablespoon of apple sauce was dispersed to modify the texture, thereby reducing the ability of subjects to discern the placebo from the test phase. The same amount of

Table 1

Demographics and relevant biochemical data for patients with NIDDM and healthy volunteers who participated in the study

\begin{tabular}{|c|c|c|}
\hline & NIDDM & Non-diabetic \\
\hline $\begin{array}{l}\text { Number } \\
\text { (Males/Females) }\end{array}$ & $5 / 5$ & $5 / 5$ \\
\hline Age (years) & $50 \pm 9(28-59)$ & $41 \pm 10(29-59)$ \\
\hline Weight (kg) & $97 \pm 22(68-138)$ & $87 \pm 18(59-113)$ \\
\hline $\begin{array}{l}\text { Body mass index } \\
\left(\mathrm{kg} / \mathrm{m}^{2}\right)\end{array}$ & $31.3 \pm 5.3$ & $30.2 \pm 6.5$ \\
\hline $\begin{array}{l}\text { Fasting blood } \\
\text { glucose }^{\text {a }} \\
(\mathrm{mmol} / \mathrm{l})\end{array}$ & $12.2 \pm 4.21$ & $5.7 \pm 0.72$ \\
\hline $\begin{array}{l}\text { Fasting insulin } \\
\quad(\mathrm{pmol} / \mathrm{l})\end{array}$ & $286.3 \pm 380.3$ & $126.3 \pm 72.5$ \\
\hline $\begin{array}{l}\text { Total cholesterol } \\
(\mathrm{mmol} / \mathrm{)})\end{array}$ & $5.10 \pm \quad 0.85$ & $6.15 \pm 0.50$ \\
\hline
\end{tabular}

Values are presented as mean \pm S.D. and/or mean (range). ${ }^{2}$ Parameters which differed significantly (as determined by unpaired $t$-test at the 0.05 level) between NIDDM patients and healthy volunteers. gelatin $(8.5 \mathrm{~g})$ was used in both the test and placebo formulations. The diet Jello ${ }^{\circledR}$ formulations were consumed during the course of a breakfast, which was served at $08: 00 \mathrm{~h}$ and consisted of $1 \mathrm{oz}$ cereal with $8 \mathrm{oz}$ milk, two pieces of toast with margarine and jam, coffee or tea and $6 \mathrm{oz}$ orange juice. This breakfast contained a total of $600 \mathrm{kcal}$ with $13.7 \mathrm{~g}$ protein ( $9 \%$ of calories), $102 \mathrm{~g}$ carbohydrates $(67 \%$ of calories) and $16 \mathrm{~g}$ fat $(24 \%$ of calories). Subjects were instructed to consume both the breakfast and the diet Jell- $O^{\oplus}$ formulation over a period of more than 10 but less than 20 min. Blood samples were drawn prior to and at 15 , $30,45,60,75,90,120,150,180,210,240$, and 360 min following the consumption of both breakfast and Jell- $\mathrm{O}^{\odot}$ with time zero taken as the time at which ingestion of both the meal and the diet Jell$\mathrm{O}^{\circledR}$ was complete. At 2-h intervals after the consumption of the breakfast, subjects were asked to complete a questionnaire regarding adverse effects including heartburn, bloating, gastric discomfort, flatulence, GI cramping, diarrhea, headache and palpitations and permitted to drink up to $8 \mathrm{oz}$ of water.

Studies in patients with NIDDM were performed on an in-patient basis. Patients reported to the GCRC the afternoon of the pre-study day, had a preselected diabetic dinner and spent the night at the GCRC. They were woken up at about $06: 15 \mathrm{~h}$ to have their catheters inserted. Following the completion of the second phase of the study they were evaluated for electrolyte balance, renal and hepatic function, and blood morphology. Studies in non-diabetic volunteers were performed on an out-patient basis with the volunteers reporting to the GCRC by $07: 15 \mathrm{~h}$ on the morning of the study days.

\subsection{Assays}

Blood glucose concentrations were measured using a Beckman ${ }^{\circledR}$ glucose analyser 2 (Fullerton, CA) which determines glucose by means of the oxygen rate method [12] employing a Beckman Oxygen Electrode. Typical precision in glucose measurements on a day-to-day basis is within 0.2 $\mathrm{mmol} / \mathrm{l}$ (standard deviation) for values in the normal range. For a $8.3-\mathrm{mmol} / \mathrm{l}$ aqueous standard, glucose measurements deviate less than $0.1 \mathrm{mmol} / 1$ 
within a run. If the sample concentration was less than $13.8 \mathrm{mmol} / 1$, determination of blood glucose was made at least in duplicate, while if the concentration was higher than $13.8 \mathrm{mmol} / \mathrm{l}$ the determination was made at least in triplicate.

A double-antibody radioimmunoassay was used for measuring plasma insulin levels [13]. The interassay and intra-assay variabilities are $6.2 \%$ and $2.0 \%$, respectively.

\subsection{Analysis of data}

For both glucose and insulin data, the maximum concentration, $C_{\max }$, and the time at which this concentration occurred, $T_{\max }$, were obtained directly from the individual blood or plasma concentration versus time profiles. The area under the concentration versus time profiles for the first $6 \mathrm{~h}$ following the consumption of the meal, $A U C_{0-6 \mathrm{~h}}$, was calculated geometrically using the trapezoidal rule [14], after excluding the area beneath the fasting level. Differences in all three pharmacokinetic parameters as well as in the concentration at each specific sampling time were assessed with repeated measures analysis of variance using the program SuperANOVA (Abacus Concepts, Berkeley, CA). The effect of the order in which the subjects participated in the two phases of the study, and the effect of the subject group (NIDDM vs. nondiabetic) on postprandial blood glucose and plasma insulin concentrations were also evaluated. In cases where variance increased with mean parameter value, the analysis was performed on $\log$ transformed data to remove this bias. The significance of the reported adverse effects was assessed with the Wilcoxon's signed rank test. In all cases differences were considered significant at the 0.05 level.

\section{Results}

Figs. 1 and 2 show the mean postprandial blood glucose and insulin concentrations versus time in the NIDDM and the non-diabetic groups, respectively. Asterisks denote statistically significant differences between phases at specific sampling times. Tables 2 and 3 summarize the pharmacokinetic analysis of the glucose and insulin concentrations in both the NIDDM patients and in the non-diabetic volunteers.

\subsection{Order of phases}

In both groups the order in which subjects participated in the two phases had no effect on either blood glucose or plasma insulin levels, indicating a lack of carry-over effect.

\subsection{Effect of HPMC in patients with NIDDM}

Blood glucose concentrations were significantly lower at the 60-, 75-, 90-, 120- and 150-min sampling times following administration of HPMC (Fig. 1A). Compared with the placebo phase, $C_{\max }$ was an average of $23.6 \%$ lower in the test phase (Table 2) and $A U C_{0-6 \mathrm{~h}}$ was reduced by an average of $14.8 \% . T_{\max }$ values were not significantly affected by the presence of HPMC.

Plasma insulin concentrations were little affected by administration of HPMC. The trend for insulin concentrations to be lower in the HPMC phase reached significance only at $120 \mathrm{~min}$. Pharmacokinetic parameters $C_{\max }, T_{\max }$ and $A U C_{0-6 \mathrm{~h}}$ were not affected (Table 3 ).

\subsection{Effect of HPMC in non-diabetic subjects}

HPMC had no significant effects on either the postprandial blood glucose concentrations (Fig. 2A) nor the glucose pharmacokinetic parameters (Table 2).

After administration of HPMC, plasma insulin concentrations were significantly lower at the 60 -, 75 - and 90-min intervals, but were higher $240 \mathrm{~min}$ after ingestion of the HPMC meal was completed (Fig. 2B). However, pharmacokinetic parameters $\left(C_{\max }, T_{\max }, A U C_{0-6 \mathrm{~h}}\right)$ for insulin in the nondiabetic group were not affected by HPMC (Table $3)$.

\subsection{NIDDM versus non-diabetic subjects}

As expected, the postprandial blood glucose concentrations were significantly higher in patients with NIDDM than in the non-diabetic volunteers throughout the sampling period (0-360 $\min$ ). Both $C_{\max }$ and $A U C_{0-6 \mathrm{~h}}$ were significantly higher in the NIDDM than in non-diabetic subjects with $T_{\max }$ occurring significantly later in the NIDDM than in the non-diabetic group (Fig. 1A vs. Fig. 2A). Repeated measures ANOVA showed that these differences between NIDDM and nondiabetic subjects were significantly greater in the 

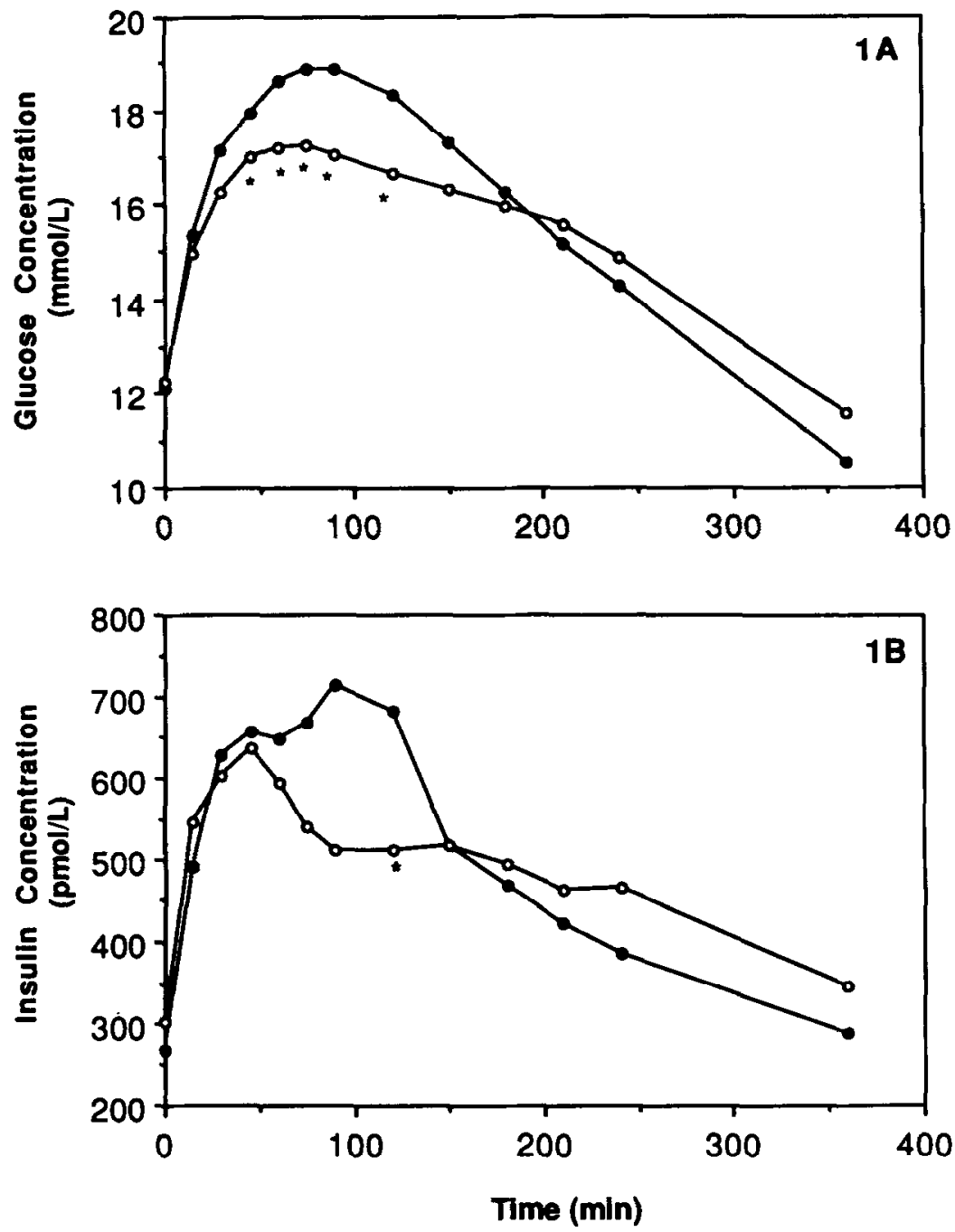

Fig. 1. (A) Mean plasma glucose and (B) plasma insulin concentrations in 10 NIDDM subjects after consuming a carbohydrate rich breakfast without (O) or with HPMC (O). Asterisks denote significant differences between treatments at a given time interval.

placebo phase than in the test phase (as determined by the Treatment $\times$ Group interaction).

Both insulin $C_{\max }$ and insulin $A U C_{0-6 \mathrm{~h}}$ were significantly lower in patients with NIDDM than in non-diabetic volunteers. Although there was a trend for plasma insulin concentrations in the NIDDM patients to be lower than in the nondiabetic volunteers, this did not reach significance at any specific sampling time. As with the glucose concentrations, $T_{\max }$ occurred significantly later in the NIDDM than in the non-diabetic group.

\subsection{Adverse effects}

In both the NIDDM and non-diabetic groups, subjects occasionally reported GI related adverse effects such as gastric discomfort or bloating. The effects were mild and not statistically different from the placebo phase.

\section{Discussion}

Hydroxypropylmethylcellulose is a semisynthetic ether of cellulose which, when dispersed in 

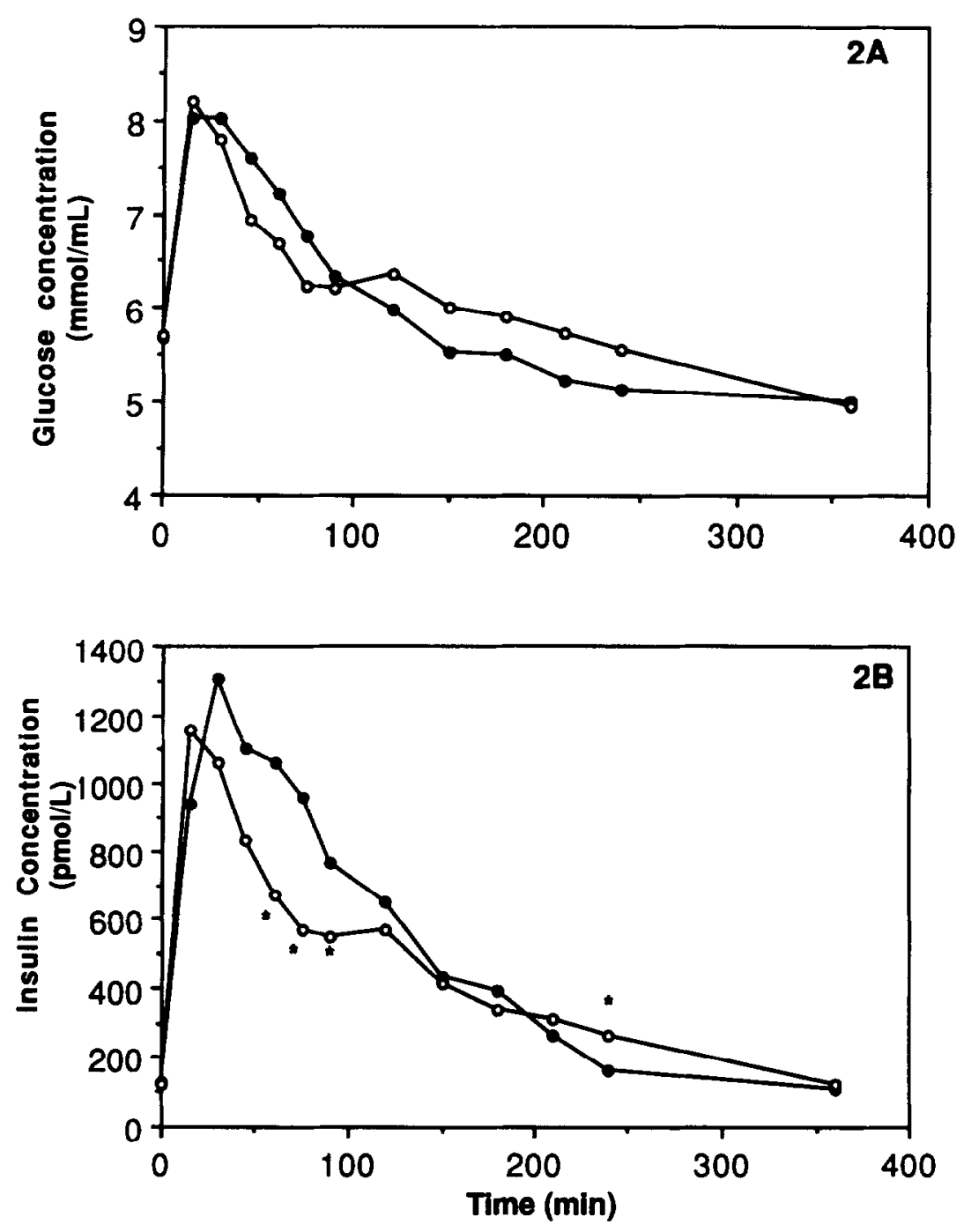

Fig. 2. (A) Mean plasma glucose and (B) plasma insulin concentrations in 10 non-diabetic subjects after consuming a carbohydrate rich breakfast without $(O)$ or with HPMC $(O)$. Asterisks denote significant differences between treatments at a given time interval.

aqueous solution, results in a striking increase in the viscosity of the solution. In this respect it is similar to naturally occurring water-soluble fibers such as guar and pectin. The viscosity achieved depends on the concentration of the polymer and its molecular weight. A $2 \%$ solution of the HPMC combination used in this study yields a viscosity of about $30000 \mathrm{cP}$ at a shear rate of $1 \mathrm{~s}^{-1}$, much higher than the viscosity of the contents of the proximal small intestine after a meal (about $40 \mathrm{cP}$ ) and comparable with that formed in solutions con- taining 3\% guar (unpublished data). Guar and pectin significantly reduce postprandial hyperglycemia and hyperinsulinemia to a similar degree, in both diabetic and healthy individuals [15]. It is therefore of interest to compare the ability of HPMC to attenuate postprandial glucose levels with published data for guar and pectin.

\subsection{Patients with NIDDM}

Seven studies in diabetic subjects have shown that when guar is administered in doses ranging 
Table 2

Effect of high viscosity HPMC on the postprandial maximum rise in blood glucose concentration, $C_{\max }(\mathrm{mmol} / \mathrm{l})$, the time that this occurred, $T_{\max }(\min )$, and the rise of the area under the concentration versus time curve, $A U C_{0-6 \mathrm{~h}}(\mathrm{mmol} \min / \mathrm{l})$, in NIDDM patients and healthy subjects

\begin{tabular}{|c|c|c|c|c|c|c|}
\hline & \multicolumn{3}{|l|}{ NIDDM } & \multicolumn{3}{|l|}{ Non-diabetic } \\
\hline & Placebo & Test & $P$ & Placebo & Test & $P$ \\
\hline$C_{\max }$ & $7.24 \pm 1.59$ & $5.53 \pm$ & 0.0099 & $2.99 \pm 1.80$ & $2.85 \pm$ & NS \\
\hline$T_{\max }$ & \pm 37 & \pm 72 & NS & \pm 10 & $21 \pm 10$ & NS \\
\hline$A U C_{0-6 h}$ & \pm 405 & $1123 \pm 298$ & 0.0096 & \pm 158 & \pm 195 & NS \\
\hline
\end{tabular}

Data are presented as mean \pm S.D.

NS indicates that differences are not significant.

from 7 to $15 \mathrm{~g}$ the maximum postprandial glucose concentration, $C_{\max }$, is reduced by percentages ranging from $2 \%$ (NS) to $61 \%$ [15]. A similar range of results has been observed with pectin. A dose of $7 \mathrm{~g}$ pectin was shown to reduce the maximum postprandial glucose concentration by $12 \%$, while $15 \mathrm{~g}$ reduced the maximum postprandial glucose concentration by $44 \%$ [15]. In the current study, $10 \mathrm{~g}$ of HPMC had an effect lower than that of the median response to guar $(53 \%)$ but comparable with that of the median response to pectin $(33 \%)$, with a reduction in the peak glucose concentration of $24 \%$.

Guar has been shown to reduce the extent of glucose absorption, as measured by the area under the curve (AUC), for the first $1-2 \mathrm{~h}$ after the meal $[1,5,6,16]$. However, when AUC was measured over longer intervals, no significant reductions were observed. We found that the AUC measured over the first $6 \mathrm{~h}$ after meal intake was reduced by an average of approximately $15 \%$ when we administered $10 \mathrm{~g}$ HPMC with the meal. These results suggest that, unlike guar [17,18], HPMC not only delays but also reduces the extent of glucose absorption. It is possible that if HPMC were administered in a less hydrated, more palatable formulation, the effects on glucose would be more modest. Even so, high viscosity HPMC appears to have two potential advantages versus other adjuvant therapies for NIDDM patients. These lie in its lack of fermentability, and therefore lesser tendency to produce flatulence [11], and, more importantly, its greater ability to control cholesterol levels [11].

Insulin concentrations tended to follow the glucose profile. There was a tendency for insulin con-

Table 3

Effect of high viscosity HPMC on the postprandial maximum rise in plasma insulin concentration, $C_{\max }$ (pmol/l), the time that this occurred, $T_{\max }(\mathrm{min})$, and the rise of the area under the concentration versus time curve, $A U C_{0-6 \mathrm{~h}}(\mathrm{pmol} \min / \mathrm{l})$, in NIDDM patients and healthy subjects

\begin{tabular}{|c|c|c|c|c|c|c|}
\hline & \multicolumn{3}{|l|}{ NIDDM } & \multicolumn{3}{|l|}{ Non-diabetic } \\
\hline & Placebo & Test & $P$ & Placebo & Test & $P$ \\
\hline$C_{\max }$ & $481 \pm$ & $390 \pm$ & NS & $1309 \pm$ & $1198 \pm$ & NS \\
\hline$T_{\max }$ & $96 \pm$ & $88 \pm$ & NS & $42 \pm$ & $30 \pm$ & NS \\
\hline$A U C_{0-6 \mathrm{~h}}$ & $77031 \pm 70552$ & $64331 \pm 44758$ & NS & $124745 \pm 85268$ & $109792 \pm 77661$ & NS \\
\hline
\end{tabular}

Data are presented as mean \pm S.D.

NS indicates that differences are not significant. 
centrations to be lower during the first $3 \mathrm{~h}$ after the meal, but higher thereafter when HPMC was administered. Differences reached significance only at the 120 -min sampling time. The similarity in the shapes of the blood concentration profiles for glucose and insulin, with tendency to a reduction in $C_{\max }$ and a delay in $T_{\max }$ suggest that glucose is gradually absorbed over a prolonged period. These results are consistent with the hypothesis that the effect of HPMC on glucose concentrations is mediated primarily via induction of a high luminal viscosity, with an associated reduction in nutrient diffusivity $[8,10]$.

The insulin results for HPMC differ from those with guar, which has a tendency to decrease postprandial $C_{\max }$ insulin levels of diabetic subjects, with reductions of $20 \%$ (NS) in one study and $68 \%$ in another [15]. Reduced GIP secretion has been considered responsible for the smaller rise in plasma insulin observed in IDDM patients when guar was added to meals [19].

\subsection{Non-diabetic volunteers}

In contrast to the response in patients with NIDDM, there was no detectable change in serum glucose concentrations when HPMC was administered with the carbohydrate-rich meal. As in the case of NIDDM patients, literature data for guar is somewhat equivocal. In 11 studies in nondiabetics, doses between 5 and $15 \mathrm{~g}$ of guar resulted in reductions in blood glucose ranging from $0 \%$ (NS) to $68 \%$, with a median of $35 \%$ [15]. In five studies with pectin, doses of $10-14.5 \mathrm{~g}$ resulted in changes ranging from an increase of $4 \%$ (NS) to a reduction of $55^{11} / 1$, with a median of $17^{\prime \prime} \%$ reduction [15]. Lack of consistency of results among studies is probably related to variations in the dose of fiber administered, the formulation given, timing of administration, carbohydrate content and subject demographics. In the current study we attempted to standardize timing of HPMC versus meal intake by asking subjects to alternate ingestion of portions of the meal with portions of the HPMC formulation. We also used a prehydrated formulation of HPMC to maximize reproducibility of the viscosity that was induced intraluminally. Another difference from previously reported studies is that we selected non-diabetic volunteers who had mildly elevated cholesterol levels as our control group, whereas other studies did not screen specifically for cholesterol. These differences in study protocol, along with the structural differences between HPMC and guar and pectin. may account for the lack of effect of HPMC on glucose concentrations observed in our control group. We attributed the absence of an effect with HPMC in our non-diabetic volunteers versus our NIDDM patients to the greater ability of insulin to control blood glucose levels in the non-diabetics.

Insulin profiles in the non-diabetic volunteers mirrored glucose concentrations as was the case in NIDDM patients. Although levels differed between phases at four sampling times (lower at 60 , 75 and $90 \mathrm{~min}$ and higher at $4 \mathrm{~h}$ ), there were no changes in the pharmacokinetic parameters. This borderline effect concurs with literature data for guar and pectin. A wide variety of effects ranging from no effect to large reductions in postprandial insulin concentrations have been observed for both guar and pectin [15]. The reduction in the postprandial insulin response to the meal that is observed for healthy volunteers when guar is added to the meal has been attributed to a reduction in GIP secretion [19].

\subsection{Comparison with canine data}

Previous studies in non-diabetic dogs indicated that co-administration of HPMC with glucose meals resulted in a $62 \%$ reduction in $C_{\max }$ and a $54 \%$ reduction in $A U C_{0-31}$, for blood glucose [8]. The discrepancy between the canine and human data might be attributable in part to the faster gastric emptying and slower small intestinal transit of non-diabetic humans compared with non-diabetic dogs [20]. The effects of HPMC on postprandial hyperglycemia seem attributable, at least in part, to reduced absorption of nutrients (impaired radial diffusion of nutrient molecules to the gut wall and delayed gastrointestinal transit $[8,10]$ ). Therefore, the slower the meal passes through the small intestine under normal meal intake conditions, the less pronounced the effect of HPMC is expected to be. A related factor would be that the AUC was calculated over a shorter interval in the case of the canine data. 


\subsection{Potential use in diabetics with hyperchol- esterolemia}

Our results indicate that addition of high viscosity HPMC to carbohydrate-rich meals results in improved control of postprandial hyperglycemia in NIDDM subjects, without significantly affecting insulin levels. A proportion of patients with NIDDM also have hypercholesterolemia [21]. In previous studies [11], we demonstrated that administration of high viscosity HPMC with meals for 1 or 2 weeks results in a reduction of total and LDL-cholesterol levels in subjects with mildly elevated cholesterol levels. A role for HPMC in the treatment of NIDDM patients who also have hypercholesterolemia, as a means of simultaneously lowering cholesterol and contributing to the control of postprandial glucose concentrations, therefore merits further investigation.

\section{Acknowledgments}

The authors wish to express their sincere thanks to Ms Cynthia Sowle (Dow Chemical Co.) who aided in the development of the formulation of HPMC, Ms Kathleen Jarvenpaa for assisting with the out-patient studies, and the GCRC nursing staff at The University of Michigan Medical Center for assisting with the in-patient studies. This research was supported by the Clinical Research Center at the University of Michigan Hospitals, Grant No. M01 RR 00042, and by funding from the Dow Chemical Company.

\section{References}

1 Wolever, T.M.S., Jenkins, D.J.A., Nineham, R. and Alberti, K.G.M.M. (1979) Guar gum and reduction of postprandial glycemia: effect of incorporation into solid food, liquid food, and both. Br. J. Nutr. 41, 505-510.

2 Jenkins, D.J.A., Goff, D.V., Leeds, A.R. et al. (1976) Unabsorbable carbohydrates and diabetes: decreased postprandial hyperglycemia. Lancet ii, 172-174

3 Jenkins, D.J.A., Leeds, A.R., Gassull, M.A., Cocher, B. and Alberti, K.G.M.M. (1977) Decrease in postprandial glucose and insulin concentrations by guar and pectin. Ann. Intern. Med. 86, 20.

4 Monnier, L., Pham, T.C., Aguirre, L., Orsetti, A. and Mirouse J. (1978) Influence of indigestible fibers on glucose tolerance. Diabetes Care 1, 83-88.
5 Jenkins, D.J.A., Wolever, T.M.S., Leeds, A.R, et al. (1978) Dietary fiber, fiber analogues and glucose tolerance: importance of viscosity. Br. Med. J. i, 1392-1394.

6 Blackburn, N.A., Redfern, J.S., Jarjis, H. et al. (1984) The mechanism of action of guar gum in improving glucose tolerance in man. Clin. Sci. 66, 329-336.

7 Pilch, S.M. (Ed.) (1987) Physiological effects and health consequences of dietary fiber. Life Sciences Research Office, FASEB, pp. 79-92.

8 Reppas, C. and Dressman, J.B. (1992) Viscosity modulates blood glucose response to nutrient solutions in dogs. Diab. Res. Clin. Pract. 17, 81-88.

9 Reppis, C., Meyer, J., Sirois, P. and Dressman. J.B. (1991) Effect of hydroxypropylmethylcellulose on GI transit and lumenal viscosity in dogs. Gastroenterology $100,1217-1223$

10 Reppas, C., Greenwood, D.E., Dermentzoglou, L.C., Meyer, J.HI and Dressman, J.B. (1992) Hydroxypropylmethylcellulose modifies the hydrodynamic responses of the canine gastrointestinal tract to nutrient solutions (Abstract). Hell. J. Gastroenterol. 5, 296.

11 Dressman, J.B., Adair, C.H., Barnett, J.L. et al. (1993). High molecular weight hydroxypropylmethylcellulose: a cholesterol-lowering agent. Arch. Intern. Med. 153, $1345-1353$.

12 Beckman glucose analyzer 2, operating manual, Beckman Instruments, Inc., Fullerton, CA, 1977.

13 Hayashi, M., Floyd, J.C., Pek, S. and Fajans, S.S. (1977) Insulin, proinsulin, glucagon and gastrin in pancreatic tumors and in plasma of patients with organic hyperinsulinism. Clin. Endocrinol. Metab, 44, 681-694.

14 Rowland, M. and Tozer, T.N. (Eds.) (1980) Clinical pharmacokinetics. Concepts and applications. Lea \& Febiger, Philadelphia, pp. 288-289.

15 Wolever, T.M.S. and Jenkins, D.J.A. (1986) Effect of dietary fiber and foods on carbohydrate metabolism. In: G. A. Spiller (Ed.). Handbook of dietary fiber and human nutrition. CRC Press, Boca Raton FL, Chapter 4.1.

16 Edwards, C.A., Blackburn, N.A., Craigen, L. et al. (1987). Viscosity of food gums determined in vitro related to their hypoglycemic actions Am. J. Clin. Nutr. 46, 72-77.

17 Seaman, J.K. (1980) Guar gum. In: R.L. Davidson (Ed.), Handbook of water-soluble gums and resins. McGrawHill, New York, Chapter 6.

18 Jenkins, D.J.A., Wolever, T.M.S. and Bacon, S. (1980) Diabetic diets: high carbohydrate combined with high fiber. Am. J. Clin. Nutr. 33, 1729-1733.

19 Morgan, L.M., Goulder, T.J., Tsiolakis, D., Marks, V. and Alberti, K.G.M.M. (1979) The effect of unabsorbable carbohydrate on gut hormones. Diabetologia 17, 85-89.

20 Dressman. J.B. (1986) Comparison of canine and human gastrointestinal physiology. Pharm. Res. 3, 123-131.

21 Vinik, A.I. and Jenkins, D.J.A. (1988) Dietary fiber in the management of diabetes. Diabetes Care 11, 160-173. 